EUROPEAN ORGANIZATION FOR NUCLEAR RESEARCH

CERN/PS/AA/81-20

\title{
BEAM OPTICS STUDIES ON THE ANTIPROTON ACCUMULATOR
}

by

B. Autin, R. Billinge, - R. Brown, G. Carron, C. Johnson, E. Jones, H. Koziol, C. Leemann*, T.R. Sherwood, S. van der Meer, E.J.N. Wilson

\author{
Paper presented at the 1981 Particle Accelerator Conference \\ March 11-13, 1981 \\ Washington, D.C.
}

*Visitor from L.B.L., Berkeley

Geneva - March 1981 
B. Autin, R. Billinge, R. Brown, G. Carron, C. Johnson, E. Jones, B. Koziol, C. Leemann*, T.R. Sherwood, S. van der Meer, E.J.N. Wilson CERN

Geneva, Switzerland

\section{Sumsary}

The CERN Antiproton Accunulator (AA) was designed to accumulate $6 \times 10^{11}$ antiprotons per day, using the stochastic cooling techique ${ }^{1}$. Its construction was completed within two years and the first beam circulated in early July $1980^{2}$. This paper describes the conceptual design of the lattice and how multipole shim corrections were applied to develop the large betatron and momentum design acceptances. We also report how a sequence of such corrections, based on optics studies with proton beams; have been applied to the point that the machine is now approaching design performance,

\section{Theoretical Concept of the AA Lattice}

The lattice is of the separated function type with 12 FODO focusing periods, each with a phase advance of $68^{\circ}$. This configuration was chosen to keep $\gamma_{t r}$ below the $\gamma$ of the $3.5 \mathrm{GeV} / \mathrm{c}$ antiprotons injected.

The superperiodicity is 2 since we require two long straight sections where $\alpha$ is zero for the momentum cooling kickers and for the in Figure 1 shows one half superperiod. The dispersion must rise rapidly: within the first $90^{\circ}$ of phase advance between septum and injection kicker, for at the in jection kicker the injected beam and the stack must be separated by a movable shutter. There are two types of bending magnet with a strength ratio $5: 8$, chosen to give the best $\alpha_{p}$ variation ${ }^{3}$.

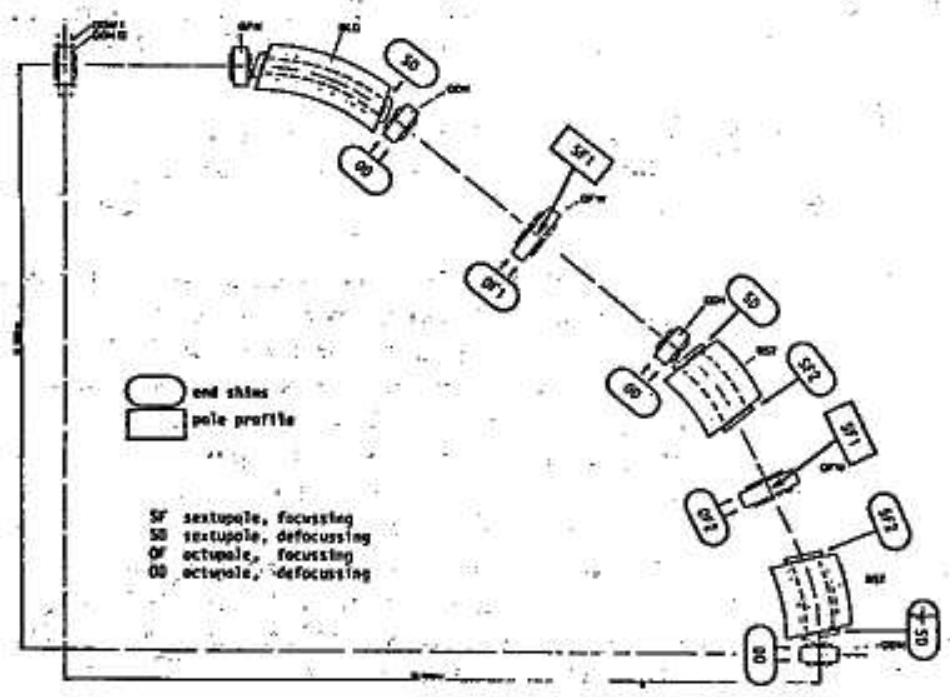

Fig. 1 - One balf superperiod of the AA ring lattice, showing where different families of shims may be applied to give sextupole and octupole correction.

Orie of the unusual features of the design is its large momentum acceptance, over $6 \%$, needed to accept 1.57 of the spectrum of $\bar{p}^{\prime} \mathbf{s}^{\prime}$ from the production target and to allow the various gymiastics of precooling, stacking and accumulation to take place at different *Visitor from L.B.L., Berkeley revolution frequencies. Another requirement is that the transverse acceptance tust be large, $100 \pi \mathrm{mm} . \mathrm{mrad}$ in each plane, to collect enough $\overline{\mathrm{p}}$ 's. To provide such large betatron acceptances yet avoid the influence of high order betatron resonances on a stored bean whose momentun is modulated first by synchrotron motion and later by cooling, is a challenging task, and it is largely this which has prompted the sequence of corrections described below. Fortunately, intensity dependent instabilities are likely to be of ninor importance in this machine and one can aim to keep the Q-spread small.

Another special requirement is that dispersion $\alpha_{p}$ must be zero within tight tolerances in the long straight section over the whole momentum acceptance to avoid transverse heating of the bean by momentum cooling. The increase of betatron amplitude in $\tau$ hours is given by

$$
\Delta a=\frac{n \dot{\alpha}_{p}}{T}\left(\frac{\dot{\gamma}}{\gamma+1}\right) \sqrt{\hat{f}_{r} P Z \tau} \approx a_{p} \sqrt{\tau / 360}
$$

where: $\mathrm{n}$ is the number of cooling cavities

$\mathrm{z}$ their impedance

$P$ the power they deliver to the beam

$T$ the kinetic energy of the $\bar{p}^{\prime} s$

$\gamma$ the relativistic mass ratio

$E_{I}$ the revolution frequency

The tolerance on $\alpha_{p}$ is $5 \mathrm{~cm}$.

Beam Measurements and Corrections

Needless to say, first beam tests with the uncorrected machine showed significant departures from design optics. Fortunately these departures had been anticipated and methods of correction prepared, as described below.

The use of two types of bending magnets to shape $\alpha$ leads to a tight tolerance on their relative strength if the orbit of the central momentum particle is to be on the axis of the long straight sections. The short bending magnets have a trim supply which is adjusted to make the average of the beam position zero at these critical locations. Computer control provides a simultaneous compensation of the average bending field to make the mean of all pick-ups zero. Radial and vertical adjustments of the position of certain quadrupoles, sefected by a "most efficient corrector" algorithm, bring peak-to-peak orbit distortions to $3.2 \mathrm{morizontal1y}$ and $1.4 \mathrm{~mm}$ vertically.

The spectrum analysers used for measuring transverse Schottky signals 5 allow rapid and automatic measurements of $\mathrm{Q}_{\mathrm{H}}$ and $\mathrm{Q}_{\mathrm{y}}$ over the whole aperture. Figure 2 shows such a measurement together with $x^{*}$, the radial position where $\alpha_{\text {p }}$ should be zero, as a function of momentum. These mealurements were made before corrections were applied. 


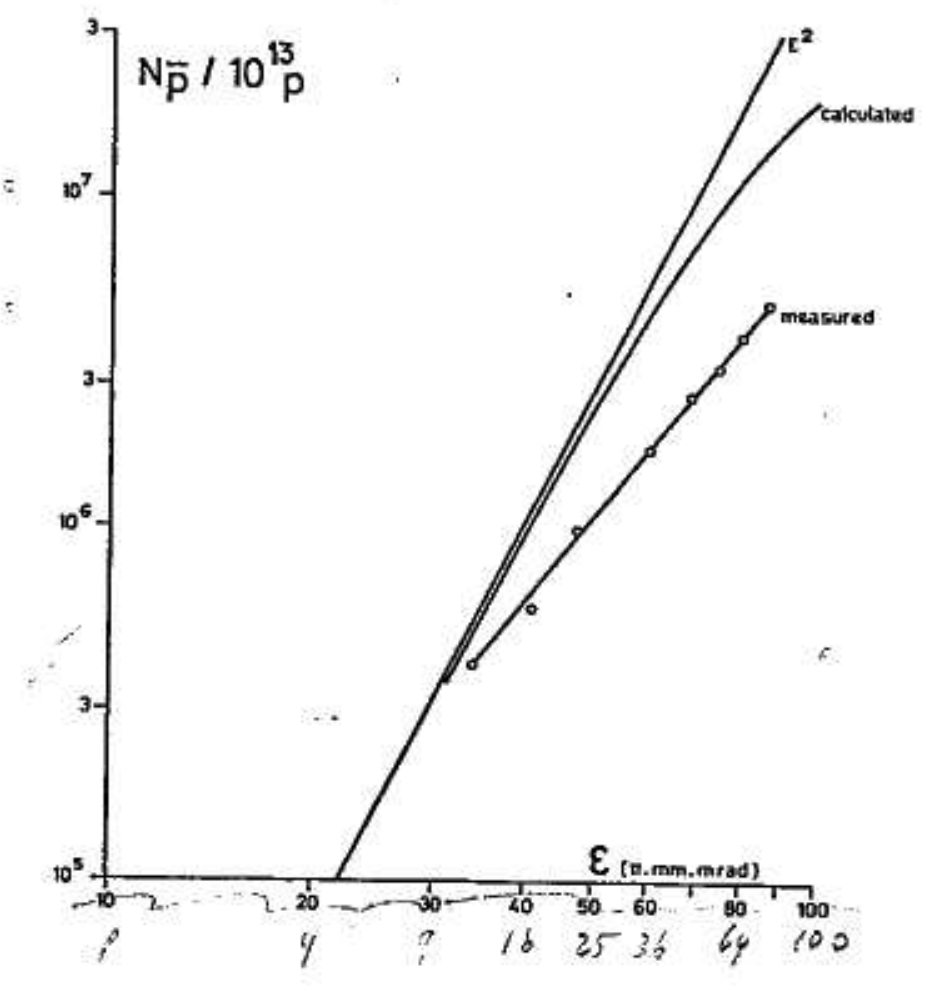

Fig. 5 - The antiprotons yield as a function of machine acceptance (emittance) by mismatching injection conditions, lost particles as it was decelerated slowly across the aperture. The losses occurred in a staircase fashion as a function of radial position. The steps in the staircase moved as the position of the working line in the $Q$ diagram was adjusted. Losses at $Q$ values corresponding to 11 th order resonances could be seen on a time scale of less than a minute when the beam emittance exceeded $40 \pi \mathrm{mm}$. mrad. The present shimming scheme brings the working line to a position which is free from resonances up to 15 th order.

Figure 6 shows the results of blowing up the emittance of the injected bean with a swept frequency applied through a transverse damping system at $(3-Q) f$. After blow up the bean is moved to another radial position and its enittances measured by moving in scrapers to touch the tails of the beam. The acceptance of the machine seems to be about $75 \pi$ mut.mrad in each plane on the injection orbit and it does not seem as if the beam meets any further acceptance limit until it is well into the stack. However, it is not certain that the beam exittance will have cooled sufficiently by the time it reaches this next restriction and we nay yet have to extend the plateau of acceptance.

The reason for the $75 \pi 1$ imit and the further constriction at the stack, whether it be a geometrical obstruction, an orbit distortion or the influence of the higher order resonances is being vigorously sought.

\section{Conclusions}

The planned procedure for improving the optics of the AA ring by applying new patterns of shims to the pole faces according to measurements with beam, has worked out well in practice and the AA ring optics is close to the precision required. Transverse acceptances are within $60 \%$ of design values and work is under way to improve then further.

\section{References}

1. R. Billinge and M.C. Crowley-Milling, The CERN Proton-Antiproton Colliding Beam Facilities, Proc. Part. Acc. Conf., San Francisco (1979).

2. J. Gareyte, The CERN p- $\vec{p}$ Complex., Int. Conf. on Bigh Energy Accelerators, CERN, July 1980.

3. B. Autin, Dispersion Suppression with Missing Magnets in a FODO Structure; Application to the CERN Antiproton Accumulator, Proc. Part. Acc. Conference, San Francisco (1979).

4. B. Autin and Y. Marti, Closed Orbit Corrections of A.C. Machines Using a Smal1 Number of Magnets, CBRN ISR-MA/73-17.

5. J. Borer, P. Bramhan, H.G. Hereward, K. Hübner, W. Schnel1, L. Thorndahl, Non Destructive Diagnostics of Coasting Beams with Schottky Noise, IV Int. Conf. on High Energy Accelerators, SLAC, May 1974, p. 53.

6. B. Autín, Simultaneous Correction of Chromaticity and Orbit Dispersion in a Strong Focussing Machine, XI Int. Conf, on High En. Acc,, CERN, July 1980.

7. D. Dekkers et al., Experimental Study of Particle Production at Small Angles in Nucleon-Nucleon Collisions at 19 and $23 \mathrm{GeV} / \mathrm{c}$, Phys. Rev. 137, B 962 (1965).

G. Bellettini et al., Proton Nuclei Cross Sections at $20 \mathrm{GeV}$, Nuc1. Phys. 79 (1966), 609.
Fig. 6 - Beam survival and emittance as the dilated injected beam is moved to different momenta 